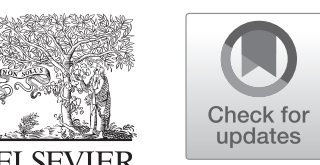

\title{
An Atypical Presentation of Thrombotic Microangiopathy After Lung Transplant: A Case Report
}

\author{
Maria do Mar Menezes ${ }^{a, *}$, Inês Aires ${ }^{a}$, Luísa Semedo ${ }^{b}$, Joaquim Calado ${ }^{a}$, \\ Francisco Ribeiro ${ }^{a}$, and Fernando Nolasco ${ }^{a}$

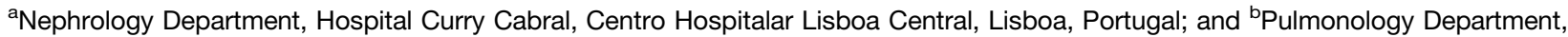 \\ Hospital Santa Marta, Centro Hospitalar Lisboa Central, Lisboa, Portugal
}

\begin{abstract}
Thrombotic microangiopathy (TMA) is a pathologic condition characterized by microangiopathic hemolytic anemia, thrombocytopenia, and organ injury due to microvascular endothelial lesions and thrombosis. It occurs in a variety of diseases and, unless recognized and treated, leads to severe morbidity and mortality. We present the case of a 48-year-old woman who underwent lung transplantation, initially under tacrolimus, mycophenolate mofetil (MMF), and prednisolone. Several complications emerged in the following months, including abdominal aortic and left renal artery thrombosis and cutaneous infections, although her renal function remained normal. Six months after transplant, her renal function began to deteriorate, which was assumed to be due to elevated tacrolimus levels and doses were adjusted. Due to leukopenia, MMF was changed to everolimus. One year after, she was admitted with fatigue, anemia, and renal dysfunction. Complementary exams revealed only iron deficiency, leukopenia, normal platelets, and elevated lactate dehydrogenase; her renal ultrasound was normal. A renal biopsy was performed and thrombotic microangiopathy was subsequently identified as the main cause of the renal dysfunction. Tacrolimus was therefore discontinued and MMF restarted with slow improvement of renal function. Only when everolimus was stopped did the patient's renal function show incremental improvement. TMA may be a serious complication after lung transplantation and the risk is higher when a combination of tacrolimus and everolimus is used. Renal biopsy findings are essential to confirm the final diagnosis of TMA, allowing for a change in immunosuppression to prevent permanent and severe renal damage.
\end{abstract}

$\mathbf{T}$ HROMBOTIC microangiopathy (TMA) is pathologic entity characterized by microangiopathic hemolytic anemia, thrombocytopenia, and organ injury due to microvascular endothelial lesions and thrombosis. It occurs in a variety of diseases and, unless recognized and treated, leads to severe morbidity and mortality [1-3]. TMA may be classified as inherited or acquired primary, secondary, or associated infection [1]. Inherited primary encompass mostly mutations in ADAMTS13; genes encoding complement proteins and cobalamin deficiency and acquired primary englobe autoantibodies to ADAMTS13 or to complement factor $\mathrm{H}$. The associated infections are mainly shiga-toxin-producing Escherichia coli and Pneumococcus. Secondary TMA occurs due to a spectrum of conditions including drugs, severe hypertension, pregnancy, glomerular diseases, autoimmune conditions, malignancies, and solid organ transplantation.
Sometimes a trigger (infection, transplantation, medication) may be required to manifest a preexisting disorder (asymptomatic mutations of the complement regulatory genes), leading to clinical manifestations of TMA [4].

\section{CASE PRESENTATION}

We present the case of a 48 -year-old woman with a past medical history of extrinsic allergic alveolitis and anemia in the context of menometrorrhagia with unknown etiology. The respiratory condition evolved into pulmonary fibrosis and chronic respiratory failure

*Address correspondence to Maria do Mar Menezes, Nephrology Department, Hospital Curry Cabral, Rua da Beneficência no8. 1050-099, Lisboa, Portugal. Tel: +351918414235 . E-mail: mariamarmenezes@gmail.com

$0041-1345 / 19$ https://doi.org/10.1016/j.transproceed.2019.05.002 
and the patient underwent a unilateral lung transplant and began immunosuppression with a calcineurin inhibitor (CNI), tacrolimus, mycophenolate mofetil (MMF), and prednisolone. She experienced complications shortly after. One month after transplant she was diagnosed with a lung infection, methicillin-sensitive Staphylococcus aureus, and treated with levofloxacin. Three months later she was admitted to hospital due to abdominal aortic and left renal artery thrombosis, pericardial effusion, and hemodynamic instability. She was treated with anticoagulants that were later maintained for 6 months. Main thrombophilias were excluded (no Leiden nor Prothrombin 20210A mutation). Her echocardiogram results were normal and her renal function remained stable at discharge (creatinine $.7 \mathrm{mg} / \mathrm{dL}$ ).

During the following months she suffered many cutaneous infections (folliculitis, facial abscess, axillar abscess), some of which resulted from the methicillin-sensitive Staphylococcus aureus infection and were treated flucloxacillin. Six months after the transplantation, her renal function began to deteriorate (serum creatinine [Scr] $1.26 \mathrm{mg} / \mathrm{dL}$ ). This fact was assumed to be linked to elevated CNI levels and therefore her doses were adjusted (Scr oscillated between 1.26 and $2.4 \mathrm{mg} / \mathrm{dL}$ ). Due to leukopenia, MMF was changed to a mammalian target of rapamycin (mTOR)-everolimus (EVE).

One year after the surgery the patient was admitted to the Pneumology Department with anemia (microcytic, hypochromic; hemoglobin $7.1 \mathrm{~g} / \mathrm{dL}$ ) iron deficiency (iron $37 \mathrm{ug} / \mathrm{mL}$ and transferrin saturation $17 \%)$; leukopenia $(2.810)$, normal platelets $\left(201 \times 10^{9}\right)$ and renal dysfunction (Scr $3.2 \mathrm{mg} / \mathrm{dL}$ and serum urea $63 \mathrm{mg} / \mathrm{dL}$ ). Apart from fatigue, there were no other clinical signs or symptoms, such as pulmonary complaints or bloody diarrhea. A renal ultrasound revealed that her kidneys were of normal size and regular contours, with slightly augmented cortical echogenicity. Proliferative lesions, excretory dilatation, lithiasis, and peri-renal collections were excluded.

The patient was transferred to the Nephrology Department for investigation. Complementary exams revealed elevated lactate dehydrogenase $(683 \mathrm{U} / \mathrm{L})$, normal haptoglobin $(.42 \mathrm{~g} / \mathrm{L})$, rare schistocytes in the peripheral blood smear, normal coagulation, negative Coombs test, normal serum protein electrophoresis, and autoimmunity (C3 $1.70 \mathrm{~g} / \mathrm{L} ; \mathrm{C} 4 \quad 30 \mathrm{~g} / \mathrm{L})$. The complement phenotype evaluation was normal (AH50 and $\mathrm{CH} 50$ functional studies were normal > 100\%); factors $\mathrm{H}, \mathrm{B}$, and I were within normal range; antibody anti factor H:9.2 UA/mL $(<27)$; and ADAMTS13 activity was $1.14 \mathrm{UI} / \mathrm{mL}(>.67)$. The patient was negative for cytomegalovirus viral load and human immunodeficiency virus were negative. Urinalysis showed proteinuria $(50 \mathrm{mg} / \mathrm{L})$ with no white or red blood cells. Tacrolimus levels oscillated between $3.57-11 \mathrm{ng} / \mathrm{mL}$ and EVE between $1.9-2.8 \mathrm{ng} / \mathrm{mL}$. A renal biopsy was performed that revealed ischemic glomerulus, endotheliosis, intracapillary and arteriolar thrombi, and stripped fibrosis (Fig 1). Thrombotic microangiopathy was then identified as the main cause of the renal dysfunction. Tacrolimus was discontinued and MMF restarted, resulting in a slow improvement of renal function (Scr $2.04 \mathrm{mg} / \mathrm{dL}$ ). Meanwhile, the patient developed severe aphthous stomatitis and everolimus was stopped, although MMF and prednisolone were maintained. After this change, renal function showed incremental improvement to Scr $1.4 \mathrm{mg} / \mathrm{dL}$. EVE was started again after 9 months at a small dosage. To date, no differences on renal function have been noted.

\section{DISCUSSION}

This interesting case reflects the importance of a multidisciplinary approach and the importance of renal biopsies in making a diagnosis. The absence of thrombocytopenia and

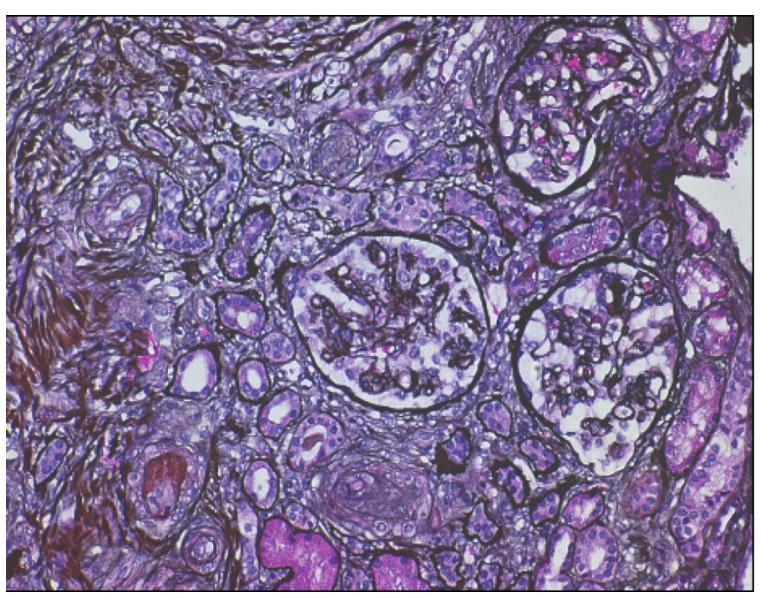

Fig 1. Kidney biopsy (silver staining, 200x) showing ischemic glomerulus, endotheliosis, intracapillary and arteriolar thrombi, and stripped fibrosis.

the preexisting anemia were confounding factors that delayed the hypothesis of TMA. Initially, the supratherapeutic CNI levels could be related to worsening of the renal function (assuming CNI nephrotoxicity), but that pattern was not present afterwards. Despite the multiple cutaneous infections, no relation was found on the clinical chart, thereby eliminating the hypotheses of infectionrelated glomerulonephritis and antibiotic-related interstitial nephritis. The renal biopsy was therefore essential to achieve the diagnosis of TMA. We then tried to exclude the main causes of TMA. Hemolytic uremic syndrome caused by shiga-toxin-producing $E$ coli seemed unlikely in the absence of diarrhea (although stool was not collected). ADAMST13 activity was higher than $10 \%$, which excluded thrombotic thrombocytopenic purpura; however, despite the patient having renal insufficiency and having undergone a transplantation (a known trigger), the absence of severe thrombocytopenia made this diagnosis improbable [1]. As the complement phenotype showed no abnormalities, the main primary causes (either inherited or acquired) of TMA were excluded. No infections were detected at the time (negative blood cultures) although no specific screening for $E$ coli $\mathrm{O} 157: \mathrm{H} 7$ serotype in stool cultures or shiga toxin detection by enzyme-linked immunosorbent assay was performed. Secondary causes were then addressed, as TMA after solid organ transplant was more likely.

The incidence of TMA after lung transplant is very low $(2.3 \%)$ [1,5] and the median time of onset is 37 weeks, as it was in our patient [5]. The pathogenesis is not completely understood. It is probably multifactorial with many different contributors, including ischemia-reperfusion injury, antibody-mediated rejection, cytomegalovirus infection, and CNI therapy [1], and is eventually complement-mediated [6]. The combination of CNI and mTOR increases the risk of TMA, rather than the use of CNI alone [7].

Treatment recommendations for TMA have changed in recent years. Besides plasma exchange, eculizumab is now 
recommended in specific cases, but in transplant-associated and drug-induced TMA, the primary treatment is still immunosuppression change $[1,3,4,8]$. In a small study of 7 patients who underwent organ transplantations (5 small bowel and 2 liver) complicated by TMA, eculizumab was used after treatment failure with CNI reduction, withdrawal, and plasmapheresis. All patients made a full recovery. Despite the unclear pathogenic mechanism, the authors assumed that CNI-induced TMA was complement-mediated and therefore amenable to eculizumab [6]. We conclude that TMA may be a serious complication after lung transplantation and that the risk is higher when a combination of CNI and mTOR is used [5]. Renal biopsy findings were essential to confirm the final diagnosis of TMA and allow immunosuppression change (CNI discontinuation), thereby preventing permanent and severe renal damage.

This case is unique not only because the incidence of TMA after lung transplant is very low, but also because the presentation was atypical. The pre-existing anemia, the absence of thrombocytopenia, a normal haptoglobin level, and only rare schistocytes made this a difficult diagnosis. Furthermore, there is no recollection of a similar case in this hospital center.

\section{REFERENCES}

[1] Brocklebank V, Wood K, Kavanagh D. Thrombotic microangiopathy and the kidney. Clin J Am Soc Nephrol 2018;13:300-17.

[2] Azevedo A, Faria B, Teixeira C, et al. Portuguese consensus document statement in diagnostic and management of atypical hemolytic uremic syndrome. Port J Nephrol Hypert 2018;32: 211-32.

[3] George JN, Nester CM. Syndromes of thrombotic microangiopathy. N Engl J Med 2014;371:1847-8.

[4] Bommer M, Wölfle-Guter M, Bohl S, et al. The differential diagnosis and treatment of thrombotic microangiopathies. Dtsch Arztebll Int 2018;115:327-34.

[5] Verbiest A, Pirenne J, Dierickx D. De novo thrombotic microangiopathy after non-renal solid organ transplantation. Blood Rev 2014:28:269-79.

[6] Broome C, McCloskey J, Girlanda R. Successful management of calcineurin-induced thrombotic microangiopathy with eculizumab after non-renal solid organ transplantation. Blood 2013;122:1078

[7] Hachem RR, Yusen RD, Chakinala MM, et al. Thrombotic microangiopathy after lung transplantation. Transplantation 2006;81:57-63.

[8] Reig Mezquida JP, Jover AS, Ansótegui Barrera E, et al. Thrombotic microangiopathy associated with tacrolimus in lung transplantation. Arch Bronconeumol 2015;51:e23-4. 